\title{
Uso de posaconazol en niños: experiencia en un hospital pediátrico de alta complejidad
}

\author{
Use of posaconazole in children: Experience in a tertiary pediatric hospital
}

\author{
Dra. María T. Rosanova a ,Dra. Carla Voto ${ }^{a}$, Dra. María S. Mussini ${ }^{a}$ Dra. Claudia Sarkis ${ }^{a}$, Dra. Sandra Gómeza \\ Farmacéutica Norma Sberna ${ }^{b}$, Dra. Susana Carnovalec, Dra. Beatriz Caracciolo y Dr. Roberto Leded
}

\section{RESUMEN}

La información sobre el uso de posaconazol en niños es escasa. Se realizó este estudio descriptivo retrospectivo entre agosto de 2010 y marzo de 2017 para evaluar las características clínicas, microbiológicas y la evolución de los pacientes tratados con posaconazol.

Se incluyeron 16 niños. Mediana de edad: 161 meses (rango intercuartílico -RIC- 69-173 m). Todos tenían enfermedad subyacente y presentaban infección fúngica invasiva probada. Los aislamientos más frecuentes fueron Mucor spp. y Aspergillus spp. La dosis media de posaconazol fue $600 \mathrm{mg} /$ día (400-800 mg / día) y la mediana de duración del tratamiento, 223 días (RIC 48-632). Diez pacientes presentaron efectos adversos, pero solo uno requirió suspensión del antifúngico debido a alteraciones hidroelectrolíticas.

Palabras clave: posaconazol, tratamiento, infecciones fúngicas invasivas, niño.

\section{ABSTRACT}

There is limited information on the use of posaconazole in children.

This retrospective and descriptive study was conducted to evaluate the clinical, microbiological characteristics and evolution of patients treated with posaconazole between August 2010 and March 2017.

Weincluded 16 children. Median age: 161 months (interquartile range-IQR-69-173 m). All had underlying disease and a proven invasive fungal infection. The most frequent isolated were Mucor spp. and Aspergillus spp. The mean posaconazole dose was $600 \mathrm{mg} /$ day (400-800 mg/day) and the median duration of treatment was 223 days (IQR 48-632). Ten patients had adverse effects, but only one required suspension of the antifungal treatment due to hydroelectrolytic disorders.

Key words: posaconazole, treatment, invasive fungal infections, child.

a. Servicio de Control Epidemiológico e Infectología.

b. Servicio de Farmacia.

c. Servicio de Micología.

Hospital "Prof. Dr. Juan P. Garrahan", Ciudad Autónoma de Buenos Aires, Argentina.

d. Universidad Abierta Interamericana (UAI), Buenos Aires, Argentina.

Correspondencia:

Dra María T. Rosanova: margris2@yahoo.com.ar

Financiamiento: Ninguno.

Conflicto de intereses: Ninguno que declarar.

Recibido: 15-9-2017

Aceptado: 22-1-2018 http: / / dx.doi.org/ 10.5546/ aap.2018.e451

Cómo citar: Rosanova MT, Voto C, Mussini MS, et al. Uso de posaconazol en niños: experiencia en un hospital pediátrico de alta complejidad. Arch Argent Pediatr 2018;116(3):e451-e454.

\section{INTRODUCCIÓN}

Las infecciones fúngicas invasivas (IFI) son frecuentes en niños con enfermedades oncohematológicas. ${ }^{1-3}$ Candida spp. y Aspergillus spp. son los hongos más comunes, aunque Cryptococcus spp., Fusarium spp. o Zygomycetes también pueden aislarse. ${ }^{4}$ En este escenario, los azoles, como el fluconazol, carecen de acción frente a hongos filamentosos y algunas especies de Candida no albicans, y las equinocandinas tienen acción reducida frente a Zygomycetes, por lo que ambos tendrían utilidad limitada. ${ }^{5}$

El posaconazol es un triazol con actividad in vivo frente a un amplio espectro de especies fúngicas, como Candida spp., Aspergillus spp., Criptococcus spp., Zygomycetes spp. y Fusarium spp., que pueden ser una alternativa en estos casos. ${ }^{1-5}$

La agencia sanitaria de los Estados Unidos (US Food and Drug Administration; FDA, por sus siglas en inglés) aprueba su uso en niños mayores de 13 años como profilaxis de IFI por Candida spp. y Aspergillus spp. en pacientes con enfermedad hematooncológica y trasplantados de médula ósea. ${ }^{2}$ La Agencia Europea de Medicamentos (European Medicines Agency; EMA, por sus siglas en inglés) lo aprueba como terapia de salvataje en IFI refractarias o en intolerantes a la terapia convencional. $^{2}$

El objetivo de este estudio es presentar las características clínicas, microbiológicas y de evolución de niños tratados con posaconazol.

\section{MATERIALES Y MÉTODOS}

Estudio descriptivo retrospectivo. Se incluyeron todos los pacientes menores de 18 años internados en el Hospital "Prof. Dr. Juan P. Garrahan" entre agosto de 2010 y marzo de 2017 que recibieron posaconazol como tratamiento de IFI. 


\section{Definiciones}

Las IFI se definieron según los criterios de la European Organization for Research and Treatment of Cancer/Invasive Fungal Infections Cooperative Group and the National Institute of Allergy and Infectious Diseases Mycoses Study Group (EORTC/MSG). ${ }^{3}$

En todos los casos, el posaconazol fue indicado como terapia de continuación para completar el tratamiento por vía oral o de salvataje en los pacientes refractarios o intolerantes a las drogas antifúngicas convencionales.

El posaconazol fue administrado como suspensión oral en una dosis de 400-800 mg/ día, dividido en dos dosis.

Se evaluaron los efectos adversos relacionados con su administración y, para ello, en todos los pacientes, se realizaron controles de hemograma, función renal, ionograma, calcemia, fosfatemia y hepatograma al inicio, durante y al final del tratamiento.

Su indicación fue aprobada por el Comité de Medicamentos del Hospital.

\section{Análisis estadístico}

Se adoptaron la mediana y el rango intercuartílico (RIC) como medidas de posición y dispersión. La significación de las diferencias $(p<0,05)$ entre los valores al ingreso y egreso se

Tabla 1. Aislamientos fúngicos

\begin{tabular}{lc}
\hline Aislamientos fúngicos & $\mathbf{N}$ \\
\hline Mucor spp. & $5 / 16$ \\
Aspergillus spp. & $2 / 16$ \\
Scedosporium spp. & $1 / 16$ \\
Rhizopus spp. & $1 / 16$ \\
Cladosporium spp. & $1 / 16$ \\
Aspergillus spp. y Candida spp. & $1 / 16$ \\
Aspergillus spp. y Alternaria spp. & $1 / 16$ \\
Aspergillus nidulans y Fusarium spp. & $1 / 16$ \\
Aspergillus spp. y Mucor spp. & $1 / 16$ \\
Aspergillus spp., Mucor spp. y Fusarium spp. & $1 / 16$ \\
Aspergillus spp., Mucor spp. y Rhizopus spp. & $1 / 16$ \\
\hline spp.: especies. &
\end{tabular}

evaluó acorde a las características de las variables, con la prueba exacta de Fisher o con la prueba de $t$, según correspondiera.

\section{RESULTADOS}

Se incluyeron 16 pacientes. La mediana de edad fue de 161 meses (RIC 69-173 m.). Todos tenían enfermedad de base: $5 / 16$, leucemia linfoide aguda (LLA); 4/16, trasplante de médula ósea; 4/16, enfermedad granulomatosa crónica; $1 / 16$, aplasia medular; $1 / 16$, trasplante cardíaco, y $1 / 16$, quemadura.

En todos, la IFI fue probada. Los aislamientos se resumen en la Tabla 1. Los sitios de aislamiento fueron los siguientes: senos paranasales y órbitas (3 pacientes), piel (3 pacientes), pulmones (2 pacientes), hígado, tejido óseo y colon (1 paciente).

Todos habían recibido terapia antifúngica previa; en la mayoría de los casos, anfotericina b o complejo lipídico (que eran las drogas de elección en nuestro centro) y 8 pacientes recibieron posaconazol con otro antifúngico.

La dosis de posaconazol tuvo una media de $600 \mathrm{mg} /$ día (400-800 mg/día) y la mediana de duración de tratamiento fue de 223 días (RIC 48-632). El dosaje de posaconazol se obtuvo en 6 pacientes; la mediana fue de $647 \mathrm{ng} / \mathrm{ml}$ (RIC 246-826).

Diez pacientes presentaron efectos adversos: trastornos hidroelectrolíticos en 6, aumento de transaminasas en 4 y alteración de la función renal en 3.

Solo 1 paciente debió interrumpir el tratamiento por hipokalemia relacionada con el fármaco.

Ninguna de las diferencias observadas en los parámetros biológicos entre los valores al ingreso y al egreso resultó diferente de cero para el nivel de confianza del 95\% ( $\mathrm{p}>0,05)$ (Tabla 2).

Cinco niños fallecieron por progresión de la enfermedad de base, no relacionada con el uso de posaconazol.

Tabla 2. Parámetros bioquímicos al inicio y al final del tratamiento

\begin{tabular}{lccc}
\hline Parámetros bioquímicos & $\begin{array}{c}\text { Comienzo del tratamiento: } \\
\text { mediana (RIC) }\end{array}$ & $\begin{array}{c}\text { Fin del tratamiento: } \\
\text { mediana (RIC) }\end{array}$ & $\boldsymbol{P}$ \\
\hline GPT $(\mathrm{UI} / \mathrm{l})$ & $55(17-200)$ & $29(16-125)$ & $\mathrm{ns}$ \\
Recuento de GB $\left(/ \mathrm{mm}^{3}\right)$ & $6630(3675-12000)$ & $5120(3855-7135)$ & $\mathrm{ns}$ \\
Hemoglobina $(\mathrm{g} / \mathrm{dl})$ & $9,3(8,3-10,3)$ & $9,9(9,3-10,9)$ & $\mathrm{ns}$ \\
Plaquetas $\left(/ \mathrm{mm}^{3}\right)$ & $119500(27500-350500)$ & $140000(40500-245550)$ & $\mathrm{ns}$ \\
Urea $(\mathrm{mg} / \mathrm{dl})$ & $26(16,5-43)$ & $24,5(15-40,5)$ & $\mathrm{ns}$ \\
\hline
\end{tabular}

RIC: rango intercuartílico; GPT: transaminasa glutámico-pirúvica; GB: glóbulos blancos; ns: no significativo. 


\section{DISCUSIÓN}

El posaconazol es un antifúngico triazólico de amplio espectro que emerge como opción para la profilaxis y/o tratamiento de IFI. ${ }^{1,2}$

Estudios clínicos en fase III han evaluado la eficacia de posaconazol como tratamiento de salvataje o mantenimiento en adultos. ${ }^{2} \mathrm{Sin}$ embargo, la evidencia disponible de su uso en niños es limitada.

En este estudio, todos los pacientes fueron inmunocomprometidos, como en la bibliografía consultada. ${ }^{6,7}$

Lehrnbecher et al. ${ }^{6}$ reportaron una serie de 15 niños inmunocomprometidos que recibieron tratamiento de salvataje con posaconazol por IFI probable o probada, con resultados favorables. Segal et al. ${ }^{7}$ evaluaron su uso como terapia de segunda línea en 8 niños con enfermedad granulomatosa crónica y $7 / 8$ alcanzaron una respuesta completa.

En la serie que se presenta, las infecciones por Mucor spp. y Aspergillus spp. predominaron, al igual que en otras series publicadas. ${ }^{6,8} \mathrm{El}$ compromiso sinuorbitario y pulmonar fue el más frecuente, en coincidencia con la literatura. ${ }^{8}$

Lehrnbecher et al. ${ }^{6}$ reportaron que la tasa de respuesta con posaconazol fue del $60 \%$, aunque todos los niños habían recibido terapia antimicótica sistémica previa y solo 6 de los 15 pacientes reportados recibieron posaconazol como monoterapia.

Todos los pacientes de esta serie habían recibido tratamiento antifúngico previo y la mitad continuaron recibiendo terapia combinada.

La indicación de posaconazol se relacionó con la falla del tratamiento previo, toxicidad o como terapia de mantenimiento. La evolución favorable fue menos frecuente que en otras series. ${ }^{6,7}$ Esto puede deberse a la gravedad de la enfermedad de base de los pacientes, a que todas las IFI fueron probadas y a que, en la mayoría de los casos, el posaconazol fue un tratamiento de salvataje.

En pediatría, los datos referidos a parámetros farmacocinéticos y farmacodinámicos de la droga son escasos. El posaconazol se comercializa en comprimidos de liberación retardada, presentación endovenosa y suspensión oral.

En este estudio, todos los pacientes recibieron suspensión oral y, si bien la absorción de la droga no pudo determinarse, ciertos factores inherentes a los pacientes, como la presencia de mucositis o diarrea, pudieron haber contribuido a la variabilidad en la respuesta individual. ${ }^{1}$
La EMA recomienda una dosis de $400 \mathrm{mg}$ cada 12 h o 200 mg cada 6 h con la coadministración de comida grasa y con una dosis máxima de $800 \mathrm{mg} /$ día en adultos. ${ }^{9}$ La dosis e intervalo en pediatría es variable.

Krishna et al. compararon los niveles plasmáticos de posaconazol en 12 niños mayores de 8 años y 194 adultos. Encontraron niveles séricos similares luego de una dosis de $800 \mathrm{mg} /$ día en intervalos variables ( $200 \mathrm{mg}$ cada $6 \mathrm{~h}$ o $400 \mathrm{mg}$ cada $12 \mathrm{~h}$ ). Esto permitió inferir que la eficacia podría ser similar en ambos grupos poblacionales. ${ }^{10}$ En menores de 12 años, una dosis $>18 \mathrm{mg} / \mathrm{kg} /$ día permitiría alcanzar concentraciones plasmáticas adecuadas. ${ }^{11}$

En esta serie, la dosis diaria administrada tuvo una media de $600 \mathrm{mg}$ / día en intervalos de 8-12 h. El dosaje plasmático de la droga se realizó en solo 6 pacientes y esto se debió a la falta de estandarización de la práctica teniendo en cuenta el amplio período en estudio. Se recomienda el monitoreo de los niveles séricos de posaconazol, que debe ser mayor de $700 \mathrm{ng} / \mathrm{ml}$ en pacientes con IFI. Los pacientes evaluados alcanzaron una mediana de $647 \mathrm{ng} / \mathrm{ml}$, algo menor al límite propuesto. No obstante, el impacto de los niveles plasmáticos de posaconazol en la evolución clínica no se ha determinado fehacientemente. ${ }^{12-15}$

Ensayos clínicos en fase III muestran que el posaconazol puede causar fiebre, diarrea, náuseas, hipokalemia y hepatotoxicidad. ${ }^{14}$

Döring y col. ${ }^{15}$ reportaron su experiencia en 33 niños, en los que evidenciaron aumento de las transaminasas, pero en ningún caso debió suspenderse el tratamiento.

En nuestra serie, los trastornos electrolíticos, como hiponatremia e hipokalemia, fueron los más frecuentes, pero no obligaron a la discontinuación del tratamiento, a excepción de un paciente.

La mitad de nuestros pacientes recibía antifúngicos concomitantes $\mathrm{y}$, por su enfermedad de base, todos se encontraban recibiendo tratamientos con potencial hepatotoxicidad, nefrotoxicidad o posibilidad de alteraciones hidroelectrolíticas, lo cual dificultaba atribuir un rol al posaconazol en el desarrollo de los efectos adversos observados.

Las limitaciones del estudio son el diseño retrospectivo con obtención de datos sujetos a la información de las historias clínicas.

Es, a nuestro conocimiento, la serie pediátrica más importante en nuestro país que sugeriría un rol del posaconazol como tratamiento de salvataje en IFI por hongos solo sensibles a dicha droga o 
en pacientes con mala evolución o intolerancia a otros antifúngicos.

Se requieren más estudios aleatorizados y controlados para confirmar estos hallazgos.

\section{REFERENCIAS}

1. Zoller E, Valente C, Baker K, et al. Development, clinical utility, and place in therapy of posaconazole for prevention and treatment of invasive fungal infections. Drug Des Devel Ther. 2010;4:299-311.

2. Clark N, Grim S, Lynch J 3rd. Posaconazole: Use in the Prophylaxis and Treatment of Fungal Infections. Semin Respir Crit Care Med. 2015;36(5):767-85.

3. De Pauw B, Walsh TJ, Donnelly P. Revised Definitions of Invasive Fungal Disease from the European Organization for Research and Treatment of Cancer/Invasive Fungal Infections Cooperative Group and the National Institute of Allergy and Infectious Diseases Mycoses Study Group (EORTC/MSG) Consensus Group. Clin Infect Dis. 2008;46(12):1813-21.

4. Fariñas MC, Fernández-Sampedro M, Armiñanzas C. Formas clínicas y tratamiento de las infecciones causadas por otros hongos filamentosos. Enferm Infecc Microbiol Clin. 2012;30(7):414-9.

5. Langner S, Staber P, Neumeister P. Posaconazole in the management of refractory invasive fungal infections. Ther Clin Risk Manag. 2008:4(4):747-57.

6. Lehrnbecher T, Attarbaschi A, Duerken M, et al. Posaconazole salvage Treatment in paediatric patients: a multicentre survey. Eur J Clin Microbiol Infect Dis. 2010;29(8):1043-5.

7. Segal BH, Barnhart LA, Anderson VL, et al. Posaconazole as salvage therapy in patients with chronic granulomatous disease and invasive filamentous fungal infection. Clin Infect Dis. 2005;40(11):1684-8.

8. Huang X, Wang F, Chen Y, et al. A multicenter, open-label study of posaconazole oral suspension in the treatment of invasive fungal infections in patients refractory to or intolerant of first-line therapy. Future Microbiol. 2012;7(2):201-9.

9. Smith W, Drew R, Perfect J. Posaconazol impact on prophylaxis and treatment of invasive fungal infections: an update. Expert Rev Anti Infect Ther. 2009;7(2):165-81.

10. Krishna G, Sansone-Parsons A, Martinho M, et al. Posaconazole plasma concentrations in juvenile patients with invasive fungal infection. Antimicrob Agents Chemother. 2007;51(3):812-8.

11. Gwee A, Cranswick N, Curtis N. Posaconazole: Promising but Problematic in Practice in Pediatric Patients. Pediatr Infect Dis J. 2015;34(6):604-6.

12. Stockmann $\mathrm{C}$, Constance JE, Roberts JK, et al. Pharmacokinetics and Pharmacodynamics of Antifungalsin Children and their Clinical Implications. Clin Pharmacokinet. 2014;53(5):429-54.

13. Dolton MJ, Ray JE, Chen S, et al. Multicenter Study of Posaconazole Therapeutic Drug Monitoring: ExposureResponse Relationship and Factors Affecting Concentration. Antimicrob Agents Chemother. 2012;56(11):5503-10.

14. Kersemaekers WM, van Iersel T, Nassander U, et al. Pharmacokinetics and Safety Study of Posaconazole Intravenous Solution Administered Peripherally to Healthy Subjects. Antimicrob Agents Chemother. 2015;59(2):1246-51.

15. Döring M, Cabanillas Stanchi K, Klinker H, et al. Posaconazole plasma concentrations in pediatric patients receiving antifungal prophylaxis during neutropenia. Med Mycol. 2017;55(4):375-84. 